\title{
Affektiv ist effektiv: Dramatische Aktivitäten als Hilfsmittel zur Erlangung einer interkulturellen Sensibilität im Fremdsprachenunterricht
}

\author{
Siegfried Boehm
}

\begin{abstract}
Zusammenfassung
Um mexikanischen Deutschlernern ein fremdkulturelles Orientierungssystem näher zu bringen, das die Körpersprache miteinbezieht und emotional verankert werden kann, plädiere ich für den Einsatz des dramapädagogischen Lehansatzes. Sie kommt den realen Sprechsituationen des täglichen Lebens näher als die Unterrichtstechniken der üblichen Fremdsprachenvermittlung und eignet sich vorzüglich dazu, nonverbale Elemente in den Sprachaneignungsprozess miteinzubeziehen. Im folgenden Beitrag wird auf die Voraussetzungen dramapädagogischen Unterrichts eingegangen und es werden Vorschläge zur Auswahl und Erarbeitung von Alltagsszenen gemacht, bei deren Inszenierung bewusst auf Eigenheiten der Zielsprachenkultur zu achten ist, die den Lernenden fremd sind. Anschließend werden Schritte zum Einüben einer ausgewählten Szene vorgestellt und es wird über die Nachbereitung dieser Szene berichtet.
\end{abstract}

\section{Interkulturelles Lernen}

Man wird heute kaum noch bestreiten, dass für eine tragfähige Kommunikation zwischen Sprecherinnen und Sprechern verschiedener Kulturen interkulturelle kommunikative Kompetenzen unabdingbar sind. Während früher nur relativ wenige Sprachenlernende in Mexiko ihre Fremdsprachenkenntnisse mit Muttersprachlern der gelernten Sprache anwenden konnten, gibt es heute mehr Möglichkeiten mit fremden Kulturen in Kontakt zu kommen. Aufgrund des NAFTA $^{1}$-Vertrags und der wirtschaftlichen Beziehungen zu den europäischen Ländern gibt es eine Zunahme von ausländischen Firmen in Mexiko. Zusammen mit Spanien stellt Deutschland für das Land den wichtigsten Handelspartner in Europa dar und steht nach den USA und Japan an dritter Stelle der Exportländer Mexikos. ${ }^{2}$ Vor allem amerikanische und europäische Firmen, davon allein rund

\footnotetext{
${ }^{1}$ North American Free Trade Agreement.

${ }^{2}$ Siehe www.schielconsulting.de (15.4.2009) 
600 deutsche Unternehmen, unterhalten in Mexiko Produktions- und Vertriebsniederlassungen, was dazu führt, dass viele Mexikanerinnen und Mexikaner in Zukunft auch im eigenen Land mit Interkulturalität im Berufsleben konfrontiert werden.

Wenn man davon ausgeht, dass die meisten Fremdsprachenlernenden derzeit das Ziel haben, sich mit Menschen einer fremden Kultur erfolgreich verständigen zu können bzw. sich in ihr zurecht zu finden, ist Lernen aus interkultureller Perspektive unumgänglich. Thomas (1988: 83) definiert interkulturelles Lernen folgendermaßen:

Interkulturelles Lernen findet statt, wenn eine Person bestrebt ist, im Umgang mit Menschen einer anderen Kultur deren spezifisches Orientierungssystem der Wahrnehmung, des Denkens, Wertens und Handelns $\mathrm{zu}$ verstehen, in das eigenkulturelle Orientierungssystem zu integrieren und auf ihr Denken und Handeln im fremdkulturellen Handlungsfeld anzuwenden. Interkulturelles Lernen bedingt neben dem Verstehen fremdkultureller Orientierungssysteme eine Reflexion des eigenkulturellen Orientierungssystems.

Nach Schmenk/Hamann (2007: 379-380) zielen interkulturelle Ansätze auf das „Verstehen“ einer anderen Kultur ab, indem diese mit der eigenen Kultur verglichen werden, was zu Empathie für andere und zum Verstehen des anderen sowie seiner Standpunkte und Ansichten führen soll. Sie geben allerdings kritisch zu bedenken, dass „Verstehen lehren“ ein unangemessenes Ziel im Fremdsprachenunterricht sein könnte und, wie viele Kritiker meinen, dass es eigentlich unmöglich sei, andere, bzw. andere Kulturen zu verstehen. Was meistens unter „Verstehen“ gemeint ist, wäre eher eine selbstgerechte Einstellung dem Anderen gegenüber. Die Einsicht in fremde Kulturen ist niemals neutral, sondern stets implizit von eigenen Perspektiven und Werten beeinflusst. Den Lernenden das Verstehen anderer Kulturen beizubringen würde eher zu kulturellen Vorurteilen als zu kultureller Sensibilität führen.

Wenn interkulturelles Lernen zu sehr auf essenzielle und allgemeine Konzepte sowie auf Differenzen gerichtet ist, so wird es schwierig einen interkulturellen Dialog herzustellen. Die Überbetonung der Kontraste,„das Eigene - das Fremde“ sowie die kontrastive Perspektive dualer Kulturen „die Lernerkultur - die Zielsprachenkultur" usw. führen dazu, dass Verhalten und Werte stereotypisiert, anstatt in der Auseinandersetzung relativiert werden (cf. Hu 1999). Es sollte somit insbesondere auch in Betracht gezogen werden, was die Kulturen gemeinsam haben. Oft verstehen wir uns besser mit jemandem, mit dem wir uns bis zu einem bestimmten Grade identifizieren können. Unterschiede innerhalb mitteleuropäischer Gesellschaften festzustellen kann unterhaltend und interessant sein, im Umgang mit außereuropäischen Kulturen würde das emphatische Differenzieren aber eher Grenzen ziehen. Je entfernter die Kulturen voneinander sind, desto mehr sollten wir auf Gemeinsamkeiten achten und weniger die Unterschiede betonen. Ein Vergleich ist meistens mit einer Bewertung verbunden, und Vorstellungen von besser und schlechter bestärken nur die schon vorhandenen Vorurteile (cf. Boehm 1999). 
Fremdsprachenlernende stellen strukturelle oder kulturelle Gegebenheiten der Zielsprache in der Regel nicht in Frage und haben die Tendenz, Werte und Normen der Zielgesellschaft als absolut anzunehmen. Die Lehrperson muss hier von Anfang an versuchen klarzustellen, dass dass Kulturen erstens als heterogen, nicht als homogen und geschlossen, und zweitens als prozesshaft und dynamisch zu begreifen sind (cf. Auernheimer 2003: 75). Erfahrungsgemäß kann man das am besten vermitteln, indem Lernende ihr eigenkulturelles Orientierungssystem analysieren und somit feststellen, dass sie sich in verschiedenen Situationen nicht alle gleich verhalten, sondern es in jeder Kultur lediglich Tendenzen zu bestimmten Verhaltensmustern gibt. Erst wenn Lernende sich ihrer eigenen kulturellen Prägung(en) bewusst werden und erkennen, dass sie die fremde Sprache bzw. Kultur aus ihrer eigenen kulturell vorgeprägten Perspektive wahrnehmen, können sie die Bereitschaft bzw. die Fähigkeit entwickeln, von ethnozentrischen Ansichten Abstand $\mathrm{zu}$ nehmen (cf. Kessler und Küppers 2008: 1). Die Lehrperson hat somit die Aufgabe, den Lernenden nicht nur performativeSprachkenntnisse und ausführliche Information über das Zielsprachenland zu vermitteln, sondern sollte sie dazu anregen, das Fremde in ihnen selbst, in ihrer eigenen Umgebung sowie in der anderen Kultur zu erforschen (cf. Frimberger 2009).

\section{Mexiko und die deutschsprachigen Länder}

Auernheimer (2003: 107) betont, dass die zentralen Störquellenfaktoren bei der interkulturellen Kommunikation auf der Beziehungsseite zu suchen sind:

Die in der einschlägigen Literatur geschilderten Beispiele für Kommunikationsstörungen, meist durch differente Kulturmuster bedingt, betreffen fast immer die Beziehung, weil jemand zum Beispiel Formen der Höflichkeit oder Tabus verletzt. Dazu kommt, dass Beziehungsbotschaften überwiegend nonverbal ausgetauscht werden. Gerade die Bedeutung von Mimik und Gestik, Blickkontakt, sprachlicher Intonation, räumlicher Distanz, um nur einiges zu nennen, ist aber ebenso hochgradig kulturspezifisch wie ,unbewusst'. Denn da sie als selbstverständlich gilt, wird sie nie thematisiert.

Er identifiziert vier Dimensionen bzw. Faktoren, von denen die Erwartungen der Kommunikationsteilnehmer bestimmt werden können:

- Machtasymmetrien

- Kollektiverfahrungen

- Fremdbilder (Stereotypen, Vorurteile)

- Differenz der Codes (Scripts, Kulturstandards). 
Wenn man Mexiko und die deutschsprachigen Länder anhand der kulturellen Dimensionen von Hall (1973: 1-19; 1976: 80-105, 133-134; 1983: 44-58, 59-77 ) und Hofstede (1980, 1991 in McEntee 1998: 527-555) analysiert, wird man leicht feststellen können, dass es trotz einer offiziell westlichen Kulturrichtung folgende Unterschiede gibt: Während die deutschsprachigen Länder in Kategorien wie niedrige Machtdistanz, hoher Individualismuswert, mittlere Unsicherheitsvermeidung eingestuft werden sowie in monochronische Kulturen mit niedriger Kontextinformation, was die Kommunikation betrifft, so sind für Mexiko außer der Dimension Maskulinität ${ }^{3}$ kulturelle Kategorien wie z. B. hohe Machtdistanz, Kollektivismus, hohe Unsicherheitsvermeidung sowie Polychronismus und hohe Kontextinformation charakteristisch. Man kann anhand solcher Kategorien weiterhin zu dem Schluss kommen, dass Mexiko und die USA kulturell weit voneinander entfernt sind und sich in naher Zukunft, trotz des zunehmenden kommerziellen und kulturellen Einflusses des nordamerikanischen Nachbarn, daran kaum etwas ändern wird.

\section{Dramapädagogische Hilfsmittel als methodischer Zugang zu interkulturellem Lernen}

Im dramapädagogischen Unterricht nutzen Lehrer und Schüler bis zu dem Grade, der ihnen möglich ist, das methodische Know-How einer Dramatikerin, Regisseurin und Schauspielerin zur Inszenierung von Lernprozessen. Im Vordergrund steht dabei nicht - wie im Theater bzw. in theaterpädagogischen Projekten - die künstlerische Qualität einer Aufführung, sondern die pädagogische Qualität von Lernprozessen (Schewe 1993: 112).

Im dramapädagogischen Fremdsprachenunterricht gibt es mehrere Möglichkeiten, um Lernprozesse zu fördern und eine positive Unterrichtsatmosphäre zu schaffen. Even (2003: 182) erwähnt z. B. die Simulation Globale,

eine Unterrichtsmethode, die echtes Handeln in imaginären Situationen im Fremdsprachenunterricht initiiert. In einer simulierten fremdsprachlichen Welt bewegen sich die Lernenden als Personen mit selbst gewählten fiktiven Identitäten, treten miteinander in Kontakt, meistern Aufgaben, lösen Probleme und werden vor allem sprachhandelnd tätig.

Die Simulation Globale ist zwar dem Rollenspiel ähnlich, indem sie die Realität nachahmt,

geht aber gleichzeitig durch breit angelegte Simulationsrahmen, in denen die Teilnehmenden keine vorgegebenen Rollen übernehmen, sondern sie vielmehr selbst kreieren, sowie durch längere zusammenhängende inhaltliche Ausgestaltungen, die sich über mehrere Tage bzw. Wochen hinziehen können, weit darüber hinaus.

\footnotetext{
${ }^{3}$ Nach Hofstede (1991: Seitenzahlen) bedeutet diese Dimension „Gewinn- und Erfolgsorientierung, Prestige und Status“ anstelle von „Lebensqualität, Toleranz“, etc. welche Eigenschaften der Dimension Feminität darstellen.
} 
Dramatische Aktivitäten fördern Empathie sowie Selbst- und Fremdwahrnehmung und zielen auf einen gruppendynamischen Prozess ab. Fremdkulturelle Phänomene werden hier nicht nur erklärt, sondern am eigenen Leibe erfahren. Erst wenn ein sprachlicher Ausdruck mit einem sinnlichen Eindruck verknüpft werden kann, nimmt er für den Lernenden Bedeutung an (cf. Schewe 1993: 67).

So betont z.B. Tselikas (1999: 28) den Einbezug des ganzen Körpers im Sprachlernprozess:

Teile des spontanen Sprechens sind auch die Gestik, Mimik, der Rhythmus, die Intonation, die Proxemik, nämlich die Art und Weise, wie man in einer Begegnung körperliche Zuwendung, Nähe und Distanz regelt. Dies sind lauter Fähigkeiten, die durch den Körper zum Ausdruck kommen und nur durch den Körper gelernt werden können. Diese Elemente sind auch kulturell unterschiedlich. Jede Kultur und jede Sprache hat ihren eigenen gestischen Ausdruck, Rhythmus, ihre Mimik und ihr Verständnis von räumlicher Nähe und Distanz. Der Sprachaneignungsprozess beinhaltet somit nicht nur das Erlernen von Vokabular, Sprachstruktur etc. sondern auch das Wissen über und die Befolgung der Konventionen, die dem Ausdruck durch nonverbale Zeichen zugrunde liegen und die die Sprache begleiten. Das Erlernen einer fremden Sprache ist also gleichzeitig ein kulturelles Lernen, das schon mit den nichtverbalen Elementen der Sprache beginnt.

An der Nationalen Autonomen Universität Mexikos (UNAM) leite ich das Projekt Dramapädagogik als Mittel zur Erlangung einer interkulturellen Sensibilität im Fremdsprachenunterricht. Anhand zehn fiktiver Alltagsszenen in Deutschland sollen Lernende eine interkulturelle Sensibilität entwickeln, die ihnen beim späteren Aufenthalt im Land der Zielsprache helfen soll, sich zurecht zu finden. Die Protagonisten sind ein mexikanisches Paar, Carlos und Olivia, die ein Stipendium für einen Studienaufenthalt in München bekommen haben und dort mit verschiedenen Alltagssituationen vertraut gemacht werden müssen.

Bei den Szenen wurde besonders darauf geachtet, dass die Situationen

- authentisch sind ${ }^{4}$ und landeskundliche Information enthalten;

- nicht mehr als 10-15 Minuten in Anspruch nehmen;

- einen dramatischen Höhepunkt enthalten;

- ein offenes Ende haben, das die Lernenden selbst bestimmen können.

\footnotetext{
${ }^{4}$ Die Szenen wurden aufgrund persönlicher Erfahrungen ausgearbeitet. Man könnte evtl. kritisieren, dass sie Fremdbilder verstärken, aber in Hinsicht auf die Bewusstmachung eines fremdkulturellen Orientierungssystems wurde mehr Wert auf typische Werte und Normen der deutschsprachigen Gesellschaft gelegt. In der Nachbesprechung der Szenen müssen diese allerdings relativiert werden und die Erfahrung zeigt, dass die Lernenden im Allgemeinen erkennen, dass sich nicht alle Mitglieder einer Gesellschaft gleich verhalten.
} 
Die vorgegebenen Dialoge müssen auch nicht auswendig gelernt werden, sondern dienen als Richtlinien. Es können auch Sprechkarten angefertigt werden, von denen die Aussagen abgelesen werden können. Die ausgewählten Szenen handeln von Wohnungs- und Jobsuche, Benutzung der öffentlichen Transportmittel, Einladungen zum Essen, Einkauf, Diskothekenbesuche, usw.

Nachdem die Kursteilnehmenden sich mit den Dialogen vertraut gemacht haben, sollen sie eine Liste der interkulturellen Aspekte der Szene aufstellen und diskutieren, was in der deutschsprachigen Kultur ${ }^{5}$ aus ihrer Sicht anders ist als in Mexiko und warum. Es ist stets wichtig, dass die Lernenden ihre eigenen Hypothesen über die Bedeutung der jeweiligen Situation aufstellen und diese interpretieren. Ihre mentalen Bilder und spontanen Reaktionen werden als Ausgangspunkt genutzt, egal wie stereotyp, unvollkommen oder klischeehaft sie möglicherweise sind. Diese inneren Vorstellungen müssen diskutiert, verglichen und differenziert werden. In dieser Weise wird eine Distanz zur eigenen Kultur erreicht, die notwendig ist, um interkulturelle Fähigkeiten zu entwickeln (cf. Fischer 1999: 29-42).

Die Lehrperson kann diese Liste dann noch vervollständigen. Wenn die Lernenden nicht nur linguistisch, sondern auch physisch die Erfahrung eines fremdkulturellen Orientierungssystems machen, sind sie besser in der Lage dieses zu verstehen. Kessler und Küppers erklären wie die Dramapädagogik ein Bewusstsein für die Körperlichkeit schaffen kann, die bei der interkulturellen Kommunikation eine wichtige Rolle spielt:

Gerade bei der interkulturellen Begegnung ist es nicht ausreichend, ein Bewusstsein für die eigene bzw. die fremde Kultur zu entwickeln, das sich auf mentale Aspekte beschränkt. Genauso wenig ist es ausreichend, dem Gegenüber eine ausschließlich ,intellektuelle Offenheit' zu demonstrieren. Vielmehr müssen sowohl die ,cultura awareness' als auch die emotionale Disposition des interkulturellen Sprechers durch eine entsprechende Körpersprache unterstützt werden. Daher ist es sowohl wichtig, ein Bewusstsein für die eigene Körperlichkeit (und die des anderen) als auch die physische Äußerung von Nähe und Distanz, von Offenheit und Verschlossenheit bzw. Voreingenommenheit zu gewinnen. Ein solches Bewusstsein lässt sich durch dramapädagogisches Arbeiten einüben, denn bei der bewegungsästhetischen Arbeit wird intellektuelle und somit oft abstrakte Kommunikation zur authentischen Kommunikation, die den Austausch sprachlicher und physischer Zeichen beinhaltet und ein Bewusstsein für Körperlichkeit voraussetzt und fördert (Kessler/Küppers 2008: 16).

\footnotetext{
${ }^{5}$ Obwohl es zwischen Deutschland, Österreich und der Schweiz auch kulturelle Differenzen gibt, sind diese im Vergleich zum lateinamerikanischen Kontext für das interkulturelle Verständnis nicht besonders ausschlaggebend, weswegen hier der Einfachheit halber generalisiert wird.
} 


\section{Vorbereitung der Szenen}

Nicht nur für Lernende, sondern auch für Lehrende sind performative Aktivitäten eine Herausforderung, was sicherlich der Grund dafür ist, dass Dramapädagogik noch nicht so im Fremdsprachenunterricht eingesetzt wird, wie es zu wünschen wäre. Im Gegensatz zum Grammatikunterricht oder Leseverstehen anhand eines Textbuches begibt sich die Lehrperson in das Risiko einer Lernsituation, die nicht vorhersehbar ist. Sie kann gut ausgehen, wenn die Lernenden mitmachen, kreativ sind und Spaß an der Sache haben, sie kann aber auch schief gehen, falls niemand Lust hat zu spielen, die Lernenden nicht die richtigen Worte finden, sich ständig hilfesuchend an die Lehrperson wenden usw. Die Anweisungen zum Rollenspiel in den Textbüchern sind oft defizitär, so dass unbefriedigende Lernsituationen vorprogrammiert sind. Die Lernenden bekommen kaum „Identifikationshilfen“; es erfolgt keine reflektierte Arbeit an der Rolle, d.h. keine reflektierte Einfühlung in die Situation bzw. Person (cf. Schewe 1993: 154). Weiterhin haben die kurzen Szenen der Textbücher keinen dramatischen Höhepunkt, Kreativität und Interesse der Lernenden bleiben von vornherein ausgeschaltet (cf. ibid. 161).

Um so einer Frustration vorzubeugen, sollten die Lernenden behutsam in die dramapädagogische Unterrichtsform eingeführt werden. Erfahrungsgemäß kann das folgende Vorgehen helfen:

- Es wird mit kurzen Warm-up-Übungen im Unterricht begonnen, die die Kursteilnehmenden damit vertraut machen, sich frei im Klassenzimmer zu bewegen.

- Es ist äußerst wichtig, eine Vertrauensbasis zwischen Lehrenden und Lernenden zu schaffen. Das kann gefördert werden, indem die Lehrperson nicht ausschließlich Regie führt, sondern eine kleine Rolle in der Szene übernimmt.

- Wenn die Lernenden Spaß an einer Aktivität haben, sollte man sich an ihrer Begeisterung freuen und nicht gleich zur nächsten Aktivität fortschreiten. Es sollte möglichst in einem entspannten Rhythmus gearbeitet werden.

- Den Lernenden muss genug Zeit gegeben werden sich gegenseitig kennen zu lernen. Das kann z.B. durch mehr Partnerarbeit oder Gruppenarbeit im Unterricht geschehen.

- Die Lernenden dürfen nie das Gefühl haben überfordert oder bloßgestellt zu werden.

Erst wenn diese Voraussetzungen erfüllt sind sollte man mit dem Inszenieren der Fremdsprache beginnen. Die Lehrperson wird hauptsächlich damit beschäftigt sein, die Handlungssituation zu rekonstruieren. Sie muss die Lernenden zum Handeln inspirieren sowie die Mitarbeit unter ihnen koordinieren und stets den Überblick über die ganze Szene haben, d.h. Autorin, Schauspielerin und Regisseurin zur gleichen Zeit sein. Schewe (1993: 330-331) bemerkt dazu: 
Die Bandbreite von Inszenierungsmöglichkeiten ist offensichtlich groß, doch die Fülle von Inszenierungsideen ist von Lernergruppe zu Lernergruppe verschieden. $\mathrm{Ob}$ der Lehrer den Teilnehmern weitgehende Autonomie bei der Vorbereitung und Durchführung einer Inszenierung zutraut bzw. inwieweit er durch entsprechende Beispiele und Vorschläge einen Inszenierungsweg bahnt, muss von Fall zu Fall entschieden werden... Welche Akzente die einzelnen Gruppen bei ihrer Inszenierung setzen, hängt davon ab, wie sie den Text verstehen. Das in der Gruppe ausgehandelte Textverständnis beeinflusst die Form, in der der Text inszeniert wird.

Verschiedene institutionelle Rahmenbedingungen müssen ebenso bedacht werden, wobei längere, über mehrere Wochen regelmäßig stattfindende Workshops vorzuziehen sind. In meinem spezifischen Fall an einer Universität in Mexiko dient der DaF-Unterricht den Studierenden vorrangig als studienbegleitende Zusatzqualifikation. Da aber im Allgemeinen noch ein Defizit an Englischkenntnissen besteht, wird dem Englischen der Vorzug gegeben; wenn die Zeit lediglich für eine Fremdsprache reicht. Zudem erscheinen sie nicht regelmäßig zum Unterricht und es ist schwierig, eine Unterrichtseinheit mit denselben Studierenden über mehrere Unterrichtsstunden durchzuführen, zumal auch ein Lehrplan mit einem Textbuch eingehalten werden muss. Aus Erfahrung kann ich aber nur bestätigen, dass man fast alle Situationen eines Textbuches dramatisieren kann, wenn sich die Lernenden einmal an dramapädagogische Verfahrensweisen gewöhnt haben und eine Vertrauensbasis zwischen ihnen und der Lehrperson besteht. Sie freuen sich dann geradezu darauf, wieder einmal eine Situation „spielen“ zu dürfen.

\section{Fünf Schritte zum Einüben der Szene „In der U-Bahn“}

Mit meinen mexikanischen Studierenden, die sich im Deutschen ungefähr auf dem Sprachniveau A2 nach den Normen des Europäischen Referenzrahmens bewegen, habe ich die Szene „In der U-Bahn“ (siehe Anhang) ausgewählt. Für Menschen aus weniger industrialisierten Ländern ist es oft schwierig, sich mit den komplexen Regeln eines differenzierten öffentlichen Transportsystems auseinander zu setzen, und das beginnt eben schon bei Ankunft in Deutschland, Österreich oder der Schweiz. Man könnte dabei kritisieren, dass die Szene eher strukturelle als kulturelle Probleme aufweist. Trotzdem fanden es meine Studierenden sehr wichtig, darüber Bescheid zu wissen. Der folgende Vorschlag zum Einüben der Szenen ist zeit-und lehrplangebunden, d.h. die Lernenden konnten die Szene im normalen Unterricht erarbeiten - im Wissen, dass diese Unterrichtsarbeit mit den allgemeinen Zielsetzungen des Kurses korrespondierte. Einen Monat lang wurden zwei Wochenstunden (von vier) zur Vorbereitung genutzt. So hatten die Lernenden nicht das Gefühl etwas für die Abschlussprüfung „verpasst“ zu haben. Als die schon erwähnten Bedingungen 
erfüllt waren, verlief die unterrichtliche Inszenierung in folgenden Schritten ${ }^{6}$ :

Die Teilnehmenden lesen die Szene leise.

Danach werden Strukturen und unbekanntes Vokabular erklärt. Die Lernenden sollen interkulturelle Aspekte selbst entdecken und angeben, was ihnen fremd erscheint. Diese werden in der Gruppe diskutiert, danach macht die Lehrperson sie noch auf weitere Aspekte aufmerksam.

Die Texte werden in Rollen gelesen, d.h. jede/r Teilnehmer/in übernimmt eine Rolle und liest sie laut vor.

Wenn es keine Fragen zur Szene mehr gibt, werden Kleingruppen gebildet, in denen die Szene zu Ende geschrieben wird.

Die Lernenden entscheiden sich für eine Version und diese wird von der Lehrperson korrigiert. ${ }^{7}$

Anschließend werden die Rollen für die Szenen verteilt. Die Lernenden bestimmen selbst, welche Rolle sie übernehmen wollen. Man kann die Rollen auch auslosen. ${ }^{8}$

Jeder Teilnehmer liest erneut seine Rolle vor, wobei auf richtige Aussprache und Intonation geachtet wird. Danach lesen sie ihre Rolle in verschiedenen Stimmungen: laut, leise, traurig, lustig, langweilig, singend usw. und gehen dabei frei im Raum herum.

Die Lernenden werden an die Identifizierung mit der ausgewählten Rolle herangeführt. Dabei werden unter anderem folgende Fragen als Ausgangspunkt genutzt: Was sagen die Personen und warum? Was fühlen sie? Wie bewegen sie sich, welche, Gesten machen sie usw.?

Die Lernenden markieren ihre Texte und schreiben sie auf Sprechkarten.

Die Texte sollen so gut wie möglich auswendig gelernt werden. Je nach Umfang, Zeit und Anzahl der Teilnehmenden können sie aber auch von den Sprechkarten abgelesen werden. ${ }^{9}$

\footnotetext{
${ }^{6}$ Die Lernenden haben das erste Mal eine Szene gespielt und hatten nur wenig Erfahrung mit dramapädagogischen Unterrichtsformen.

7 Eine Gruppe ließ Carlos und Olivia davon rennen als die Tür des Waggons aufging. Da gerade Fasching gefeiert wurde, gab es viele verkleidete Leute und der Kontrolleur konnte sie unter den Maskierten nicht wieder finden. Die zweite Gruppe tauschte den älteren Herrn für eine ältere Dame aus, die sich schließlich bereit erklärte die Strafe zu zahlen und die beiden auch noch zum Essen einlud. Hier kommt es natürlich mehr auf die Kreativität der Lernenden als auf eine realistische Situation an. Die Studenten entschieden sich für diese Version, wobei am Ende bei den Erklärungen zu interkulturellen Aspekten über ihre Wahrscheinlichkeit diskutiert wurde.

${ }^{8}$ Als Lehrperson möchte man natürlich gerne bestimmte Rollen den dafür geeigneten Lernenden geben, aber ich habe die Erfahrung gemacht, dass die Teilnehmenden verantwortlicher für ihre Rollen sind, wenn sie sie selbst ausgewählt haben. Man sollte den Studierenden dabei so viel Autonomie wie möglich lassen, auch wenn man nicht immer ganz glücklich über die Rollenverteilung ist. Ich übernahm dabei auch eine kleine Rolle, was Vorteile für die Gruppendynamik mit sich bringt. Dadurch wird die Integration gefördert und das Vertrauen zwischen Lernenden und Lehrenden verstärkt. Allerdings darf die Lehrperson in dieser Phase die Szene nicht unterbrechen, um zu korrigieren. Die dramatische Handlung sollte sich entfalten können und Fehler können im Anschluss an die Szene besprochen werden.

${ }^{9}$ Die Sprechkarten waren eine Stütze für die Lernenden und wurden in der Szene zumeist geschickt versteckt.
} 
Die verschiedenen Orte der Szene werden im Klassenzimmer festgelegt, wobei Tische und Stühle zurechtgerückt, Plakate auf die Wände geklebt, die Tafel beschrieben wird, usw. ${ }^{10}$

Die Szene wird zum ersten Mal gespielt. Die Lehrperson versucht den Lernenden eine Stütze zu sein, d.h. sie sagt ein, hilft, hat eine erzählende Funktion, usw. ${ }^{11}$ Nach der Szene werden die Fehler erklärt und Hinweise auf nonverbales Verhalten gegeben.

Wenn die Szene gut eingeübt ist und es keine Einwände von Seiten der Lernenden mehr gibt, wird sie gefilmt. Dazu verkleiden sich die Teilnehmenden und können sich somit besser in die Rolle einbringen. ${ }^{12}$

Jede Teilnehmerin und jeder Teilnehmer erhält eine CD von der Aufführung.

\section{Nachbereitung der Szene}

Filmaufnahmen eignen sich besonders gut zur Besprechung und Evaluierung der Szenen. In der darauffolgenden Reflexionsphase zeigten wir die Videoaufnahme, damit alle Teilnehmer an der Szene die Möglichkeit hatten sich zu beobachten. Dabei konnte auch das nonverbale Verhalten der Lernenden analysiert und zwischen der mexikanischen und deutschsprachigen Körpersprache verglichen werden. Es versteht sich von selbst, dass Fremdsprachenlerner nicht das Verhalten von Personen der Zielsprachenkultur annehmen müssen, sie müssen es aber kennen und verstehen, damit keine Missverständnisse aufkommen. Natürlich konnten die Lernenden die deutsche Körpersprache nicht aus dem Text erkennen, aber durch die Videoaufnahme wurden sie auf ihre eigenen Verhaltensweisen aufmerksam. ${ }^{13}$

In dieser Phase nahmen wir auch eine linguistische Fehlerkorrektur in der Gruppe vor und ich machte auf gravierende Fehler aufmerksam. Auch möchte ich an dieser Stelle erneut betonen, dass die verfilmte Szene nicht perfekt sein muss. Eine perfekte Aufführung der Szene würde in einem Fremdsprachenunterricht nicht nur zu viel Zeit in Anspruch nehmen, sondern auch unnötigen Stress bei den Lernenden hervorrufen.

In der Schlussphase bat ich die Teilnehmenden der Szene um Feedback und Kommentare zu unserer Aufführung. Dazu meinten sie Folgendes ${ }^{14}$ :

\footnotetext{
10 Ich brachte einen U-Bahnplan von München sowie Fotos von der U-Bahn mit ins Klassenzimmer. So konnten sie u.a. sehen, wie ein Fahrkartenautomat aussieht, was ihnen beim Entwurf ihres eigenen für die Szene geholfen hat.

${ }^{11}$ Die Kursleiterin kann Gedanken der Personen aussprechen. Diese können auch auf einem Schild geschrieben stehen. Die Teilnehmenden fanden es auch lustig, die Gedanken auf ihren Handys aufzunehmen. Man lässt dabei am besten die Lernenden selbst entscheiden, welches Vorgehen sie vorziehen.

12 Man merkte deutlich, dass die Studenten ihre Hemmungen verloren, sobald sie verkleidet waren. Die Verkleidung hilft bei der Einfühlung in die Personen.

13 Zum Beispiel hielt Olivia die alte Dame, die ihnen die Strafe zahlte, am Arm und sagte „Oma“ zu ihr. Hier wurde diskutiert, ob eine ältere Dame in Deutschland das als liebevoll (wie evtl. in Mexiko) oder als zu dreist und fast beleidigend interpretieren würde.

${ }^{14}$ Die Diskussion über die Szene fand auf Spanisch statt. Ich machte mir dabei Notizen und
} 
- „Die CD mit der Aufführung der Szene war eine „Belohnung“ und ein Andenken an den Sprachunterricht. Sie erhöhte die Motivation und wir fühlten uns verantwortlich für die Szene.“

- „Mir hat besonders die Dekoration des Klassenzimmers gut gefallen. Dabei hatten wir viel Spaß und haben uns besser kennen gelernt.“

- „Ich fand es toll, dass wir Sprechkarten benutzen konnten, so musste ich nicht so viel Zeit damit verbringen, die Rolle auswendig zu lernen. Man durfte manches auch etwas anders sagen."

- „Falls ich einmal in ein deutschsprachiges Land kommen sollte, werde ich mich sicherlich an unsere Szene erinnern, wenn ich eine Fahrkarte aus einem Automaten holen muss. Jedenfalls habe ich schon eine Ahnung, wie das geht, kenne die verschiedenen Modalitäten und weiß, worauf ich aufpassen muss."

- „Erst als wir uns etwas verkleidet hatten, konnte ich mich in meine Rolle hineinversetzen und es war sehr lustig."

- „Ich fand es gut, dass auch unser Lehrer mitspielte, er machte auch Fehler wie wir und ich fühlte mich dadurch freier und nicht so beobachtet."

- „Meine Familie und Freunde waren ganz stolz auf mich, als sie die Videoaufnahme sahen und mich Deutsch sprechen hörten. Ich war selbst etwas darüber erstaunt und fühle mich jetzt sicherer als vor unserer Szene.“

\section{Schlussfolgerungen}

Das ständigeEinüben und Wiederholen der Rolle hat auch dazu beigetragen, dass die Lernenden ihre linguistischen Fähigkeiten verbessern konnten. Besonders Aussprache, Intonation und fließendes Sprechen wurden mehr geübt als im normalen Sprachunterricht. Die vorgegebenen Dialoge beinhalteten außerdem viele typische deutsche Redewendungen, Partikeln und umgangssprachliches Vokabular, das die Lernenden besser behalten konnten, da sie es in einem Kontext lernten, der annähernd der Realität im Zielsprachenland entspricht.

Das relative Verhalten der deutschsprachigen Gesellschaft können die Lernenden allerdings erst nach dem Inszenieren mehrerer Szenen einigermaßen nachvollziehen. Zwischen dem Eigenen und dem Fremden erreichen sie eher einen „dritten Ort“. Nach Kessler und Küppers (2008: 221) kann durch dramapädagogisches Arbeiten eine Situation entstehen, in der authentische Erfahrungen möglich sind und Lernen im Sinne eines sich Veränderns durch

übersetzte die Kommentare auf Deutsch. 


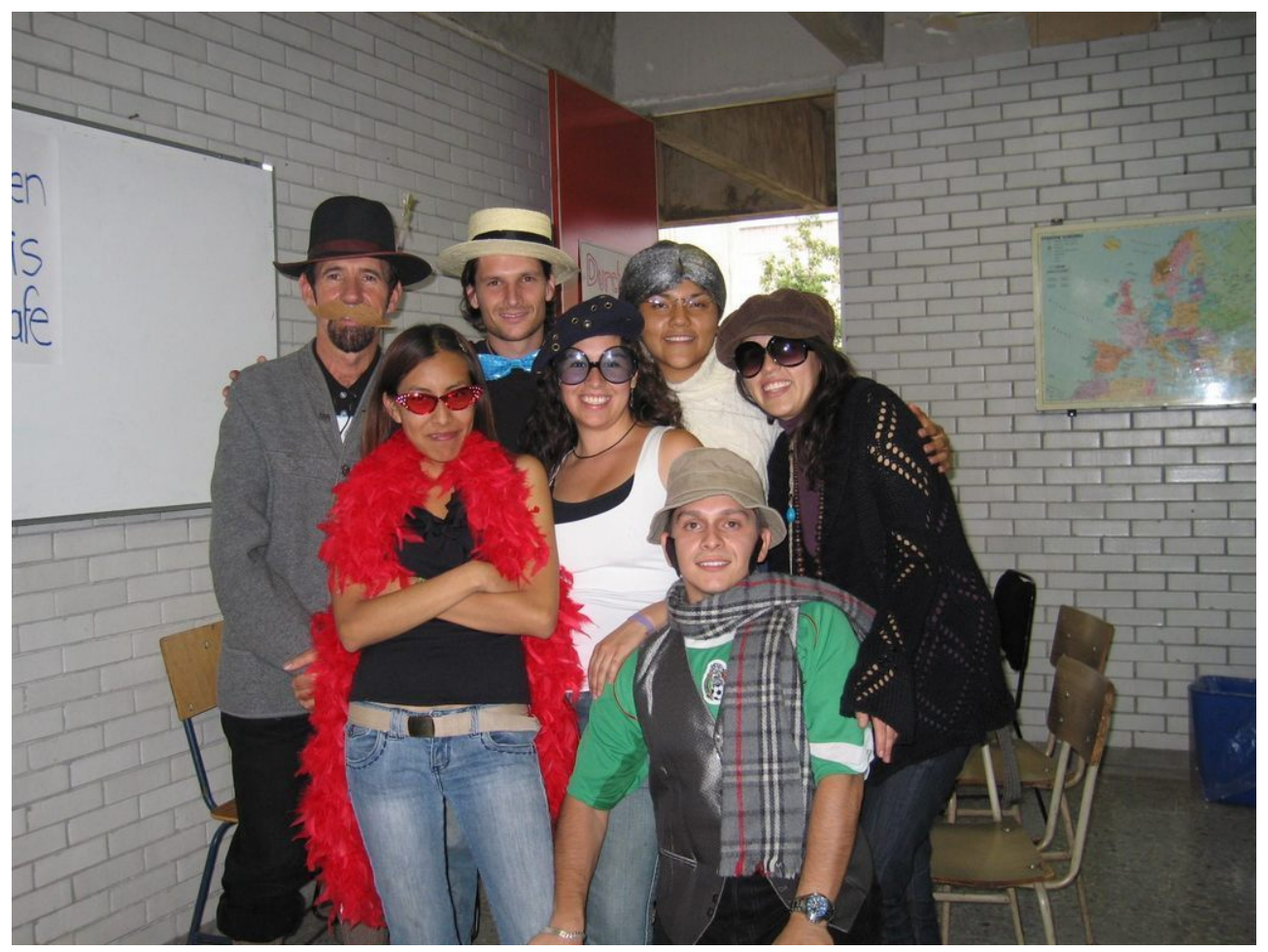

Abbildung 1: Gruppenfoto

Zweifel, Nachdenken und Überlegen geradezu notwendigerweise eintritt. Der Interkulturellen Dramapädagogik wohnt somit das Potenzial inne, den viel zitierten „dritten Ort“, die Perspektive zwischen ,dem Eigenen' und ,dem Fremden', im Fremdsprachenunterricht zu realisieren und auf interkulturelle Kompetenz in all ihren Facetten hinzuarbeiten.

Wenn auch nicht alle Fahrkartenautomaten gleich sind, so erwarben die Teilnehmer der Szene doch Wissen über ein differenzierteres Transportsystem als im Heimatland. Auch das nonverbale Verhalten konnte besser trainiert und mit der eigenen Kultur verglichen werden. Besonders Kenntnisse über Gestik, Körperhaltungen, Sprechlautstärke, Distanz zwischen den Personen, Kleidung, Begrüßungs- und Verabschiedungssrituale ${ }^{15}$, etc. wurden durch das dramapädagogische Vorgehen besser verankert.

Auffallend positiv erschien mir vor allem die Gruppenintegration. Die Teilnehmenden lernten sich durch die dramatischen Aktivitäten immer besser kennen und hatten auch außerhalb des Unterrichts Kontakt zueinander.

Es reicht also nicht aus, Werte, Normen und Verhalten einer fremdkulturellen

\footnotetext{
${ }^{15}$ In der Szene verabschiedeten sich z. B. zwei Passantinnen mit einem Kuss auf die Wange. Die Gestik eignete sich zur Erklärung, dass man sich in den deutschsprachigen Ländern eher mit der Hand begrüßt und verabschiedet und weniger mit einem Wangenkuss oder Umarmung, so wie es in Mexiko üblich ist. Obwohl man dieses Wissen oft voraussetzt, ist es für viele Lernende nicht selbstverständlich.
} 
Gesellschaft zu erklären. Die Lernenden können diese eher erfassen, wenn sie in einen Kontext eingebettet sind, der der Wirklichkeit nahe kommt. Um am effektivsten zu diesem „dritten Ort“, dem Ort der Vermittlung zwischen Eigenem und Fremdem, an dem ,aus beiden Polen ,fremd' und ,eigen' bzw. ,unbekannt und ,bekannt" etwas Neues, etwas Gemeinsames entsteht" (Kessler 2008: 90 in Schewe 2009: 3) zu gelangen, wird ein dramapädagogisches Vorgehen empfohlen - insbesondere wenn der Unterricht nicht im Zielsprachenland stattfindet.

\section{Bibliographie}

Auernheimer, Georg (2003): Einführung in die interkulturelle Pädagogik. Darmstadt: Wissenschaftliche Buchgesellschaft

Boehm, Siegfried (1999): “Los objetivos 'subjetivos' de la enseñanza de la cultura y civilización en el marco del aprendizaje de idiomas extranjeros". In: Antología del $10^{\circ}$ Encuentro Nacional de Profesores de Lenguas Extranjeras. México, D.F.: CELE, UNAM, 259-270

Even, Susanne (2003): Drama Grammatik. Dramapädagogische Ansätze für den Grammatikunterricht Deutsch als Fremdsprache. München: iudicium

Fischer, Roland (1999): „European Concepts for global intercultural communication. Can they work?" In: Antología del $10^{\circ}$ Encuentro Nacional de Profesores de Lenguas Extranjeras. México, D.F.: CELE, UNAM, 29-42

Frimberger, Katja (2009): Towards a pedagogy of strangeness. Exploring the potential of strangeness for foreign language education. In: Scenario III/1, 17-28

Hall, Edward (1973): The Silent Language. Garden City, N.Y.: Anchor Press/Doubleday

Hall, Edward (1976): Beyond Culture. Garden City, N.Y.: Anchor Press/Doubleday

Hall, Edward (1983): The Dance of Life, Garden City, N.Y.: Anchor Press/Doubleday

Hofstede, Geert (1980): Culture's Consequences: International Differences in Work-Related Values. Beverly Hills, California: Sage

Hofstede, Geert (1991): Cultures and Organizations: Software of the Mind. New York: McGraw Hill

Hu, Adelheid (1999): Interkulturelles Lernen. Eine Auseinandersetzung mit der Kritik an einem umstrittenen Konzept. In: Zeitschrift für Fremdsprachenforschung 10, 277-303

Kessler, Benedikt; Küppers, Almut (2008): A Shared Mission. Dramapädagogik, interkulturelle Kompetenz und holistisches Fremdsprachenlernen. In: Scenario II/2, 3-24 
Kessler, Benedikt (2008): Interkulturelle Dramapädagogik. Dramatische Arbeit als Vehikel des interkulturellen Lernens im Fremdsprachenunterricht. Frankfurt/Main: Peter Lang

McEntee, Eileen (1998): Comunicación Intercultural. México, D.F.: McGraw Hill

Schmenk, Barbara/Hamann Jessica (2007): "From History to Memory: New Perspectives on the Teaching of Culture in German Language Programs". In: Lorey, Christoph/Pleus, John/Rieger, Caroline (Hrsg.): Interkulturelle Kompetenzen im Fremdsprachenunterricht, Intercultural Literacies and German in the Classroom. Tübingen: Narr

Schewe, Manfred (1993): Fremdsprache inszenieren. Oldenburg: Carl von Ossietzky Universität

Schewe, Manfred (2009): Interkulturelle Dramapädagogik von Benedikt Kessler (Rezension). In: Scenario III/1, 64-67

Thomas, Alexander (1988): Interkulturelles Lernen im Schüleraustausch. Saarbrücken: Verlag für Entwicklungspolitik

Tselikas, Elektra (1999): Dramapädagogik im Sprachunterricht. Zürich: Orell Füssli 


\section{Anhang 1: In der U-Bahn}

- Mitspielende: 5-8 Personen

- Materialien und Artikel: Plakate, Stifte (Fahrkartenautomaten und Schilder malen); Tirolerhut, Trachtenjacke (Bayer); Fahrkarten, evtl. Spielgeld, Brille, Stock (älterer Herr).

\section{Szene 1: Vor dem Fahrkartenschalter}

Carlos und Olivia haben heute ihren ersten Tag an der Universität in München. Sie nehmen die U-Bahn am Sendlinger Tor und suchen einen Schalter, um die Fahrkarte zu kaufen.

Olivia: Hier verkauft wirklich niemand Fahrkarten und Polizisten gibt es auch nicht, die man fragen könnte. Ich glaube, wir sollten einfach so die U-Bahn nehmen. Man kommt ja auch ohne Fahrkarte rein.Carlos: Das ist mir zu riskant! Schau mal, hier ist ein Automat. Probieren wir's doch mal, die Fahrkarte hier zu kaufen.Olivia: Schaut den Automaten an. Oh je, da gibt es so viele Erklärungen und nur auf Deutsch! Frag doch mal, ob uns jemand da eine Fahrkarte rausholen kann.Alle rennen durcheinander.

Carlos: (denkt) „Hier hat wirklich niemand Zeit.“Entschuldigung...Passant: Ich spreche kein Deutsch. Er geht weiter.Olivia: Oh, Verzeihung, könnten Sie uns bitte behilflich sein? Wir möchten zwei Fahrkarten zur Universität.Passantin: $\mathrm{Hm}$. Das ist keine Kurzstrecke mehr. Also die Einzelfahrscheine kommen Ihnen ziemlich teuer, da würde ich Ihnen einen 10 Fahrten-Schein oder eine Tageskarte empfehlen. Wie viele Zonen brauchen Sie denn normalerweise?Carlos: Oh, wir wohnen in der Lindwurmstraße.Passantin: Da reicht die Innenzone.Ein Bayer, der schon länger zugeschaut hatte, mischt sich ein.

Bayer: Wenn Sie drei Tog' in Minchn bleim, dann nehmen'S doch die 3 Tageskarte.Olivia: Wir bleiben ein ganzes Jahr hier.Passantin: Ja, dann gehen Sie am besten zur Zeitkartenzentrale, da gibt es Wochen- und Monatskarten.Bayer: Sie brauchn bloß a Buidl und an Nachweis über Wohn- und Arbeitsort.Carlos: (wird ungeduldig) Das haben wir jetzt aber nicht dabei. Wir wollen erst mal nur eine Fahrkarte.Passantin: So, dann drücken wir einfach mal auf die 3 Tageskarte. Mit der können Sie so oft Sie wollen im Innenraum der Stadt herumfahren und es gibt auch eine für 2 Personen, wenn Sie immer zusammen bleiben.Olivia: Wir hätten lieber jeder eine.Carlos und Olivia geben der Passantin das Geld und sie holt die Fahrkarte aus dem Automaten.

Carlos und Olivia: Vielen Dank!Passantin: Bitte, gern geschehen. Sie geht weiter.Carlos: Die war aber sehr nett! Nur sind wir jetzt etwas zu spät dran. Du weißt doch, dass Pünktlichkeit hier so wichtig ist.Szene 2: Auf der Rolltreppe

Beide nehmen die Rolltreppe und stellen sich nebeneinander. Ein Passant schubst Carlos und rennt die Rolltreppe hinunter.

Carlos: Hey, ist der aber unhöflich!Olivia: Schau doch, die stehen alle rechts und gehen links. Das muss hier eine Regel sein.Olivia und Carlos gehen nach der Rolltreppe in die falsche Richtung.

Passant: Hier dürfen Sie nicht durch! Da steht doch "Durchgang verboten".Olivia: (denkt) „Was geht den das an? In Mexiko gehen wir doch immer 
durch, wo ,no pase' steht, da kommt man meistens schneller zum Zug“.Olivia und Carlos: Danke.Szene 3: Im Zug

Beide nehmen jetzt den Zug und setzen sich hin.

Carlos: Hier bekommt man wenigstens einen Sitzplatz. In Mexiko-Stadt muss man immer stehen, da die Waggons meistens überfüllt sind.Ein älterer Herr mit Stock kommt herein und wendet sich an Carlos.

Älterer Herr: Stehen Sie bitte auf, junger Mann, dieser Platz ist für Senioren reserviert.Carlos schaut verwundert und steht auf. Ein anderer Mann, ganz normal gekleidet, streckt Carlos und Olivia einen Ausweis hin.

Kontrolleur: Ihre Fahrausweise bitte!Carlos: (denkt) „Gut, dass wir nicht schwarz gefahren sind“. Bitte sehr.Kontrolleur: Ihre Fahrausweise sind nicht gültig!Olivia: Warum denn nicht? Wir haben sie doch gerade erst gekauft.Kontrolleur: Ja, aber sie sind nicht entwertet. Die Fahrkarte ist erst gültig ab Entwertung. Ich kann ja nicht wissen, wann Sie sie gekauft haben. Carlos: Sieht auf ein Schild im Wagon: Öhne gültigen Fahrausweis 60 Euro Strafe". (denkt) "Oh je, und wir haben doch nur 15 Euro dabei“.Wie geht die Geschichte zu Ende? 